Gazi University
Journal of Science
http://dergipark.gov.tr/gujs

\title{
Day-ahead Management of Energy Sources and Storage in Hybrid Microgrid to reduce Uncertainty
}

\author{
Prakash Kumar K. ${ }^{1,2}$ (D), Saravanan B.,*(iD) \\ ${ }^{1}$ Department of Technical Education, Government of Andhra Pradesh, Government Polytechnic for Women, Palamaner, India-517408. \\ ${ }^{2}$ Vellore Institute of Technology, Vellore, Tamil Nadu, India, 632014.
}

\section{Highlights}

- An Energy Management Algorithm is proposed for day-ahead scheduling in a microgrid.

- Optimization problem is framed to minimize the cost of power generation under DMO model.

- Artificial Fish Swarm technique is used as a tool to solve the optimization part.

- Several scenarios are drawn to treat uncertainty in Renewable Energy availability.

\begin{tabular}{l} 
ArticleInfo \\
\hline \\
Received:17/01/2019 \\
Accepted:25/06/2019 \\
Keywords \\
\hline Microgrid \\
Uncertainty \\
Storage \\
Energy Management \\
Day ahead scheduling
\end{tabular}

\section{INTRODUCTION}

The economic research forecasts that the cost of energy generation from RESs will nose dive next decade in comparison with their conventional counterparts like coal [1]. In realization of these forecasts, solar generated electrical energy has witnessed a lowest bidding rate of 2.44 Rupees in India from a private producer [2]. It is comparatively much cheaper than conventional sources like nuclear or coal. Apart from cost of energy generation, the potency of solar generated energy in meeting the global energy needs with almost zero damage to the environment is yet another advantage. The sunshine rich parts of the world like Asia, Africa, and Australia etc have enough solar energy sources to meet a major share of global energy requirements. The idea of tapping solar and wind energy which are distributed sources by availability has encouraged the concept of connecting smaller and distributed generation sources to the utility grid at tail ends (distribution systems) at low and medium voltages levels. This lead to a whole new concept of autonomous microgrids [3]. The microgrids operate in either grid connected mode or in autonomous mode. The developments in microgrid enabling technologies like capacity building of DG units from a few $\mathrm{kW}$ to MW, efficient energy derivation methods from RESs, reducing costs of energy storage, availability of wide area controlling technologies like SCADA along with incentives offered by governments for renewable energy generation have grown so attractive that they can no more be overlooked by investment policy makers. The investment policies in energy market are being reframed to accommodate microgrids into the existing utility grid system and symptoms that conventional centralized generation concept of energy is slowly shifting towards the distributed generation concept are evident [4]. 
All the above advantages being on one side of the microgrids, they suffer from quite a few operation and control difficulties. Uncertainty of energy availability from the RESs is one such serious setback, which ultimately affects the reliability of the system, if not treated properly. The energy output from a RES generator is not under the control of the microgrid operator. He can only curtail the generation in the event of excess generation. A lot of research is inspired by the need to reduce the uncertainty of energy availability from RESs and to balance the generation with the loads in the microgrids. The classical energy balance techniques fall short in microgrid environment due to the uncertainty in generation and loads added with bi-directional power flows, which add even more difficulty in energy balancing. Thanks to research, it has come out with quite a few modern techniques modelled and tested to contain the uncertainty. Day ahead scheduling using data mining, maintaining battery storage back-ups and spinning reserves, demand side management tools like load shifting, load curtailing (load shedding under deficit generation), demand response etc top the list. A sample of literature using the above energy balance techniques to solve various types of objective functions include mixed integer linear programming problems [5-7], linear programming problems [8]. Different types of linear programming techniques and nature inspired swarm intelligence algorithms are employed to solve the problems of optimum energy exchange (charging/discharging) schedules of batteries/storage facility [9-11]. All the above energy balancing techniques are from the generation side. The other energy balancing techniques from demand side are also tested successfully, like load shifting and shedding [12], demand response [13-16]. Load shedding being a technique used to manage the constant power loads (They are either supplied with rated power or else completely disconnected), using electric springs is a new technique to supply constant energy loads with reduced power [17]. Apart from this quite a large number of models are proposed and tested to represent the uncertainty of energy availability with the RES generators and the loads [18-21].

The problem of scheduling power generation sources and storage devices while optimizing the cost of power generation from sources is considered in this article. The solution to the problem needs two aspects to be solved. One is to find the optimal mix of generation from different sources to minimize the cost of generation and the other part is to schedule the required power among generation sources following the optimized mix as evaluated. Until late, the cost of power generation from renewable sources is considered as zero on the pretext that they need zero expenditure towards the fuel and hence they are treated as nondispatchable sources $[22,23]$. This assumption is too remote from practical reality and simplifies the optimization problem too much. A few works reported in literature have considered the cost of power generation from renewable sources as non-zero. Authors in [24] have considered cost of generation from wind and PV units as quadratic functions. Authors in [25] have proposed a new cost function for renewable power generation by computing a penalty amount for differential power between actual powers generated and forecasted power. This case is specifically proposed for the microgrid operators who do not have sufficient facilities to store all the excess energy available with renewable sources. The penalty amount so computed is made a component in computing the cost of renewable energy generation. The authors in [26] put an end to general flow of concept in literature that the power generation from renewable sources in uncontrollable. They have proposed an Automatic Generation Control strategy to control power generation from wind farms. We have considered both the conventional and renewable generators in dispatchable category with respective cost functions for both. The popular optimization techniques like GA and PSO, which are the favourites in literature, suffer from serious drawbacks in terms of computational complexities and time. More constrained the problem is, more will be these complexities. For instance, the process of fine tuning the computational parameters like fitness normalization, mutation rate, crossover parameters etc in GA is more trial and error based. Such complexities are quite lesser in number in Artificial Fish Swarm (AFS) algorithm. Though it suffers from the disadvantage of not using the past experience of the swarm members in evaluating the next step in search, AFS algorithm has the outweighing merits of higher accuracy, lesser computational time, faster convergence, very few parameters to be tuned etc. As such, AFS algorithm is used in this paper to find solution for the optimization part of the problem. The problem is framed with an objective to reduce the cost of energy generation in presence of uncertain energy availability from the RES generators under dynamic pricing of energy. A bunch of renewable and non-renewable energy sources are considered along with battery back-up. The uncertainty with RESs energy availability and loads is treated using a proven uncertainty model. The battery is charged from the excess energy available and is used to back-up supply under deficit generation. The microgrid is operated in grid connected mode with an upper limit for power drawl. The problem formulation is done with a view to 
include demand side management which allows load shedding under power shortage condition, compensating the consumer for the inconvenience caused due to power disconnection. Artificial Fish Swarm Algorithm is employed to work out the optimization part of the objective function. The problem is solved over hourly intervals for 24 hours.

\section{ARTIFICIAL FISH SWARM ALGORITHM}

The fishes in a swarm are seen continuously changing their positions. For an observer, their movements appear random and unsynchronized. But in reality, the fishes do it purposefully and with a high sense of synchronization. They move continuously to adjust their position in synchronization to the path leading to a location of their objective. Their objective could be anything like staying protected from a predator's attack, avoiding collision with neighbouring fellow fishes, finding a location where chances of finding food is better etc. Such movements of the fishes are modelled and the AFS algorithm is framed [27]. It frames a localized searching method for each of the individual artificial fish and each search ends with updating the solution to obtain the best location for the objective chosen. Each individual fish in the swarm is termed as artificial fish. Each instantaneous position that these artificial fishes occupy on their path to the objective location, symbolizes a possible solution to the problem at hand. These instantaneous positions are modelled differently for different objectives of movement by fish. The fishes exhibit different movement patterns for different behaviours like random searching behaviour, trail following behaviour, preying, swarming and mating behaviours etc. The AFS algorithm adopts these entire behaviours one after the other in its quest for the optimum solution. The AFS algorithm can be summed up as follows.

Let the current location of an individual artificial fish be

$X_{i}=\left(x_{i 1}, x_{i 2}, \ldots . . x_{i n}\right)$,

where $i$ signifies the number of control variables and $n$ signifies the total number of individual artificial fishes living in the swarm.

Then the consistency of fulfilling its objective at the location will be

$Y_{i}=f\left(X_{i}\right)$.

On its way to the location where the consistency of fulfilling objective is best, the artificial fish alters its position to a new location $X_{i}^{*}$ which is given by

$X_{i}^{*}=X_{i}+\operatorname{rand}() * \operatorname{step} * \frac{X_{j}-X_{i}}{\left\|X_{j}-X_{i}\right\|}$,

where rand ( ) is any random number that lies between ' 0 ' and ' 1 ', step is the largest radial displacement through which the fish can navigate in one move and $X_{j}$ is anyone of the calculated positions which exists within the visual extent of the fish. The calculated position $X_{j}$ is a function of behaviour of the artificial fish and it is different for different behaviours.

1. Chasing behaviour: In this behaviour, the artificial fish simply follows the fish in its front. Condition is that the position of the neighbouring fish exists within its visual extent. In this behaviour, the position $X_{j}$ is defined as

$X_{j}=$ The position of the neighbour fish.

2. Swarming / gathering behaviour: In this behaviour the artificial fish tries to move close to the centre of the swarm to ensure that it is surrounded by the fellow fishes in all directions to avoid any potential attack/predator from outside. In this behaviour, the position $X_{j}$ is defined as $X_{j}=$ $X_{i}+$ rand ( ) $*$ step $* \frac{X_{c}-X_{i}}{\left\|X_{c}-X_{i}\right\|}$

where the geometrical centre location of the swarm is denoted by $X_{c}$.

3. Foraging / Preying behaviour: In this behaviour, the artificial fish estimates the probability of finding food at different locations either by vision or sense. It searches for a location with better food consistency and directly moves in that direction. The position $X_{j}$ in this behaviour is defined as 


$$
X_{j}=X_{i}+\operatorname{rand}() * \text { visual }
$$

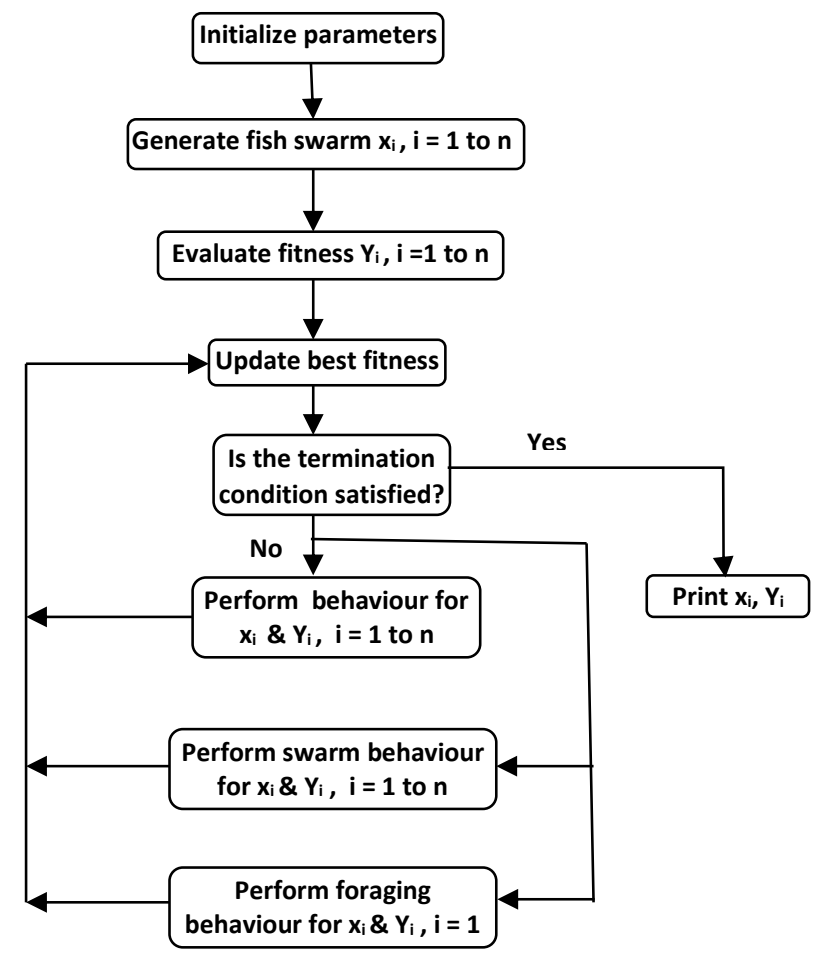

Figure 1. Flow chart of AFS algorithm

The AFS algorithm uses all the above explained behaviours of fish movement in a sequential order in search for the optimality of the objective function. With each step, the better location is updated and the search is continued until the objective is achieved. Figure-1 shows the flow chart of the AFS algorithm. After initialization of the parameters, the algorithm generates the initial fish swarm for generator $x_{i}$ for $i=1$ to $n$ as shown in Figure-1. The different artificial fishes are tested for the fitness of the objective function. The various behaviours of the fish swarm like chasing the trail, swarming and foraging behaviours, are simulated in a sequence on the artificial fishes generated as shown in Figure 1 and the best resulting locations are updated with each iteration. If the termination condition is true, the simulation is stopped.

The parametric mapping between the AFS algorithm and the problem considered is done as follows. The power generation to be scheduled to each generator is mapped with artificial fish, the vision of the fish is mapped with maximum power limit of the generator and the step of the fish is mapped with generation ramp allowed with the individual generators.

\section{MODELLING OF RELEVANT AGENTS}

The mathematical models of different agents used in this problem such as generators, storage devices, cost of energy, uncertainty associated with the loads and renewable energies availability etc are detailed as below.

\subsection{Generator model}

Let $P_{i}^{\text {con }}(t)$ and $P_{j}^{\text {res }}(t)$ be the powers generated by $i^{\text {th }}$ conventional generator (non-renewable energy) and $j^{\text {th }}$ renewable generator at time interval $t$. The upper and lower limits of their power generation are given by 


$$
\begin{aligned}
& P_{i}^{c o n_{\text {min }}} \leq P_{i}^{c o n}(t) \leq P_{i}^{c o n_{\text {max }}}, t \in T, i \in I, \\
& P_{j}^{r e s_{\text {min }}} \leq P_{j}^{r e s}(t) \leq P_{j}^{r e s_{\text {max }}}, t \in T, j \in J .
\end{aligned}
$$

\subsection{Storage model}

Battery storage is modelled as a distributed unit and their charging and discharging schedule is controlled by the energy management system based on the following constraints.

Let $P_{k}^{s t g_{c h}}(t), t \in T, k \in K$ and $P_{k}^{s t g_{d c h}}(t), t \in T, k \in K$, be the charging and discharging powers respectively.

Then the rates of charging and discharging are bound by upper and lower limits as in (8) and (9)

$$
\begin{aligned}
& 0 \leq P_{k}^{s t g_{c h}}(t) \leq P_{k}^{s t g_{c m_{\text {max }}}}, t \in T, k \in K, \\
& 0 \leq P_{k}^{s t g_{d c h}}(t) \leq P_{k}^{s t g_{d c h} \text { max }}, t \in T, k \in K, \\
& \operatorname{SoC}_{k}^{\text {min }} \leq S o C_{k}(t) \leq S o C_{k}^{\text {max }}, t \in T, k \in K, \\
& \operatorname{SoC}_{k}(t+1)=\operatorname{SoC}_{k}(t)+\eta_{k} P_{k}^{s t g_{c h}}(t), t \in T, k \in K, \\
& \operatorname{SoC}_{k}(t+1)=\operatorname{SoC}_{k}(t)-\eta_{k} P_{k}^{s t g_{d c h}}(t), t \in T, k \in K .
\end{aligned}
$$

The magnitude of energy stored in the battery storage facility is expressed in terms of state of charge, $S_{o} C_{k}(t)$ which could either be in terms of percentage of full charge or in terms of $\mathrm{kWh}$ remaining for backup. It is convenient to use kWh remaining for back-up in the present case. The state of charge is bound by upper and lower limits (10). The state of charge at any instant is given by (11) while charging and by (12) while discharging, inclusive of charging and discharging efficiencies as noted in equations.

\subsection{Load model}

A constant power model is used for loads. In such models the loads are an aggregate of rated powers, which should be supplied in full. A controllable/load shedding version of the model allows the energy management system to either supply the load fully or disconnect it from the grid. The load in excess to total power available with the microgrid cannot be supplied and is disconnected/ shed. Such load is termed as load shed $L_{m}^{\text {shd }}(t)$ [28]. The load shedding is allowed with compensation $C_{m}^{s h d}(t)$ due to the energy user by the utility operator for the inconvenience caused.

The Distribution Market Operator (DMO) model [29], which is used in the present work, restricts power transactions between the utility grid and microgrid to a predetermined award, which is communicated to the microgrid operator by the DMO one day in advance. The aim of this restriction is to reduce penetration of uncertainty from microgrids into the utility grid. This award is binding on the microgrid operator and he has to draw power from the utility grid without deviating from this award. Any deviation from the award will be penalised. In other words, the DMO decides the upper and lower limits for power drawl from the utility grid and utility grid is no more an infinite source. Under such conditions, if the load to be supplied by the microgrid is more than the sum of all internal sources, storage and maximum grid power limit, the excess load is disconnected and is termed as load shed.

\subsection{Modelling of costs of energy}

The business operation of microgrid involves various components towards the calculation of cost of energy production. Such components which are considered in the present article may be summed up as follows.

$$
C_{i}^{c o n}(t)=X_{i}^{c o n}(t) * c_{i}^{c o n}(t) * P_{i}^{c o n}(t)+U_{i}^{c o n}(t) * c_{i}^{c o n} \text { start }+V_{i}^{c o n}(t) * c_{i}^{c o n} \text { shut }, i \in I, t \in T,
$$




$$
\begin{aligned}
& C_{j}^{r e s}(t)=X_{j}^{r e s}(t) * c_{j}^{r e s}(t) * P_{j}^{r e s}(t)+U_{j}^{r e s}(t) * c_{j}^{r e s_{\text {start }}}+V_{j}^{\text {res }}(t) * c_{j}^{\text {res }} \text { shut }, j \in J, t \in T, \\
& C_{k}^{\text {stg }}(t)=Y_{k}^{\text {stg }}(t) * c_{k}^{\text {stg }} * \eta_{k} * P_{k}^{\text {stg }}(t), \\
& C_{m}^{\text {shd }}(t)=Y_{m}^{\text {shd }}(t) * c_{m}^{\text {shd }}(t) * L_{m}^{\text {shd }}(t), \\
& C^{g r d}(t)=c^{g r d}(t) * P^{g r d}(t) .
\end{aligned}
$$

The hourly cost of energy generation $C_{i}^{\text {con }}(t)$ by the conventional energy source including the startup and shutdown costs is given by (13) and that of RES generator $C_{j}^{r e s}(t)$ is given by (14) which include their startup and shutdown costs. $U$ and $V$ are state vectors which give the shut-down or start-up status of the sources. A ' 1 ' is used to indicate if the generator is either started or shut down at the time interval and a ' 0 ' is used to indicate if the generator is already OFF state or is already in ON state.

The cost of power exchange from battery/storage device $C_{k}^{s t g}(t)$ is modelled as given by (15), where $Y_{k}^{s t g}(t)$ is a state vector signifying the type of power exchange of the battery/storage. $Y_{k}^{s t g}(t)$ models the three types power exchange of the $k^{\text {th }}$ battery/storage with the microgrid at time $t$. The three states are charging under excess generation, discharging under deficit generation and idle states. The energy management system schedules battery charging under excess generation and discharging under deficit generation. The term $\eta_{k}$ refers to efficiency while charging and discharging of the battery/storage facility.

The cost of load shedding by the microgrid operator is given by (16). $Y_{m}^{s h d}(t)$ is a state vector signifying ON/OFF state of the $m^{\text {th }}$ load at time $t$. A ' 0 ' in the vector signifies an ON state and a ' 1 ' signifies OFF state. Compensation cost adds to the total cost of microgrid operation in case the load shedding is done by the microgrid operator. The term $c_{m}^{\text {shd }}(t)$ is the rate of compensation payable and $L_{m}^{s h d}(t)$ is the load magnitude disconnected from the microgrid by the utility operator.

The microgrid is operated in grid connected mode. Under DMO model, the power drawl from the grid is subject to upper and lower limits and the cost of power drawl $C^{g r d}(\mathrm{t})$ is laid down by (17). The grid power comes at a dynamic price of $c^{g r d}(\mathrm{t})$ per unit energy.

\subsection{Modelling uncertainty of RES generation}

Let $P_{j}^{r e s}(t)$ be the power developed by $j^{\text {th }}$ RES generator at time interval $t$ and vector $\Psi_{j}$ be a vector that collects all the values of $P_{j}^{r e s}(t)$ for $t \in T$. Then (18) consolidates this relation

$\Psi_{j}=\left[P_{j}^{r e s}(1), P_{j}^{r e s}(2) \ldots \ldots . P_{j}^{r e s}(T)\right]$, over the time horizon $t \in T$.

To model the uncertainty of power $P_{j}^{r e s}(t)$ over the time period $t \in T$, it is assumed that the value of $\Psi_{j}$ is unknown and lies in an uncertainty set $\Psi$. Let there be an exclusive uncertainty set for each of renewable generators over the time period $t \in T$ and another exclusive uncertainty set for the total renewable power $\sum_{j, s} P_{j}^{r e s}(s)$ over a time sub-horizon $s \in T$ and say all these uncertainty sets form the subsets of the main uncertainty set $\Psi$. Then (19) relates all the above uncertainty sets,

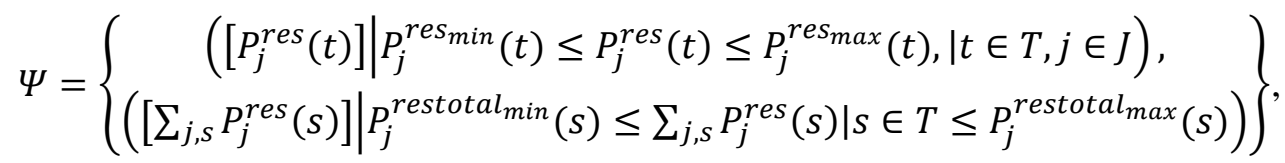

$P_{j}^{r e s_{\min }}(t)$ and $P_{j}^{r e s_{\max }}(t)$ are the minimum and maximum bounds for the values of power available with the $j^{\text {th }}$ RES sourced at time interval $t, P_{j}^{\text {restotal }_{\min }}(s)$ and $P_{j}^{\text {restotal }}$ max $(s)$ represent the minimum and maximum bounds for the values of the total energy available with the RES generators over a time subhorizon $S$. The numeric values of $P_{j}^{r e s}(t)$ and $\sum_{j, s} P_{j}^{r e s}(s)$ lie always within these respective bounds.

This uncertainty model is quite general in nature and it takes into consideration the climatic parameters of the RES sources as the model is based on the recorded data. This is a simple model in terms of computational complexity and is quite proven practically as it is based on the actual historical data. It 


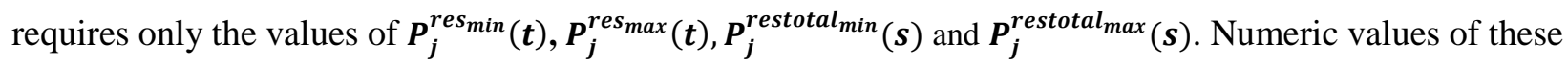
terms can be estimated from historical data analysis using any of the data inference techniques reported in literature [3].

\section{MICROGRID PROBLEM FORMULATION}

Minimization of energy generation cost being the prime objective of the microgrid utility operator, it can be stated as in (20)

$$
\min \sum_{n=1}^{N} \sum_{t=1}^{T} f\left[P_{n}(t)\right]
$$

where

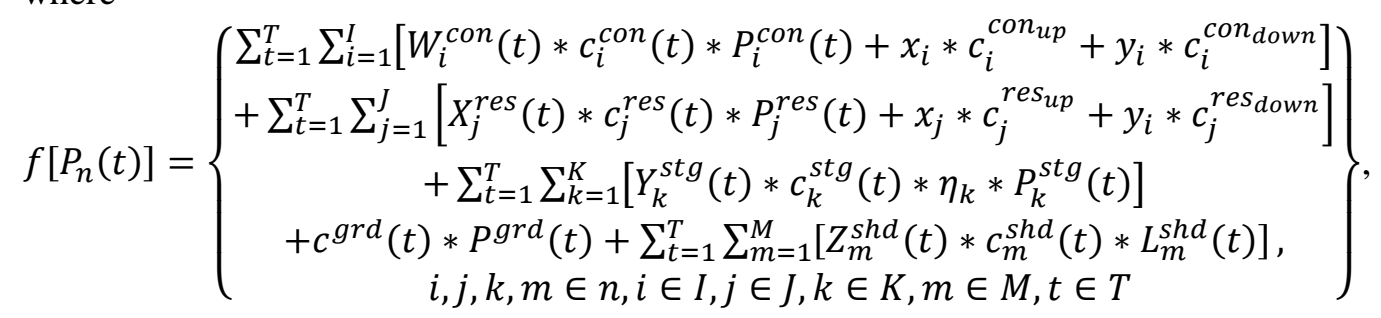

subject to the following constraints.

\subsection{Power balance constraints}

$P^{\text {generated }}(t)=P^{\text {required }}(t)$

where

$$
\begin{aligned}
& P^{\text {generated }}(t)=\sum_{i=1}^{I} P_{i}^{\text {con }}(t)+\sum_{j=1}^{J} P_{j}^{\text {res }}(t)+\sum_{k=1}^{K} P_{k}^{s t g} d c h(t)+P^{\text {grd }}(t), \\
& P^{\text {required }}(t)=\sum_{m=1}^{M}\left[L_{m}(t)-L_{m}^{\text {shed }}(t)\right]+\sum_{k=1}^{K} P_{k}^{s t g}(t), i \in I, j \in J, k \in K, m \in M, t \in T .
\end{aligned}
$$

Equation (22) is the power balance equation.

\subsection{Generator constraints}

$$
\begin{aligned}
& P_{i, t_{\text {min }}}^{\text {con }} \leq P_{i, t}^{\text {con }} \leq P_{i, t_{\text {max }}}^{\text {con }}, i \in I, t \in T, \\
& P_{j, t_{\text {min }}^{r e s}}^{\text {res }} \leq P_{j, t}^{r e s} \leq P_{j, t_{\text {max }}}^{r e s}, j \in J, t \in T .
\end{aligned}
$$

Equation (23) refers to minimum/maximum power generating limits of conventional generators whereas (24) refers to that of RES generators.

\subsection{Storage facility constraints}

The limits of battery/storage power exchange with the microgrid while charging and discharging are given by (25)

$0 \leq P_{k}^{s t g_{c h}}(t) \leq P_{k}^{s t g_{c h} \text { max }}, 0 \leq P_{k}^{s t g_{d c h}}(t) \leq P_{k}^{s t g_{d c h} \max }, k \in K, t \in T$.

The battery is charged from excess energy available. This constraint for the charging of the battery may be modelled as (26)

$$
\begin{aligned}
& P_{k}^{s t g_{c h}}(t)>0 \mid\left\{P_{i}^{\text {con }}(t)+P_{j}^{r e s}(t)+P^{g r d}(t)\right\}>\left[L_{m}(t)-L_{m}^{\text {shed }}(t)\right] \text { and } P_{k}^{\text {stg }} \text { ch }(t)=0 \mid \text { else } \\
& S o C_{k, t}^{\min } \leq S o C_{k}(t) \leq S o C_{k, t}^{\text {max }}, t \in T, k \in K
\end{aligned}
$$


Equation (27) restricts the state of charge of the battery/storage facility.

\subsection{Grid power drawl constraints}

$P^{g r d}(t) \leq P^{g r d} \max$.

Equation (28) imposes limits on power drawl from the grid and restricts it to the DMO imposed award, as explained in section 3.3 .

\section{ALGORITHM FOR PROPOSED ENERGY MANAGEMENT SYSTEM}

The proposed algorithm for day ahead energy management is implemented in following steps.

1. Read data and initialize.

2. Generate scenarios of uncertainty for RES generators and loads.

3. For each scenario

a. If total generation available is more than loads

i. $\quad$ Find power required $=$ load $+P_{\text {charge }}$.

ii. Implement AFS algorithm and schedule generation.

iii. Charge the battery with excess power available and update state of charge.

b. If the total generation is less than loads

i. Find load shedding $=$ load $-\left(\right.$ Sum of all generation available $\left.+P_{\text {dischrge }}\right)$

ii. Implement load shedding.

iii. Find power required $=$ load-loadshedding

iv. Use all the available generation

v. Discharge the battery to meet load and update state of charge

4. Evaluate cost of generation

5. Save data and end.

The objective of the proposed Energy Management Algorithm (EMA) is to schedule the generation and storage in a microgrid to reduce the effect of uncertainty in RES power availability and loads. Optimization of cost of generation is only a part of proposed EMA. The proposed EMA calls the Artifical Fish Swarm (AFS) algorithm when there is a chance to optimize the cost of generation in step 3.a. Optimization is possible only during excess generation, i.e., when the generation is more than the load. On the other hand, during deficit generation i.e., when the generation is less than the load, all the sources should be used and hence there is no chance for optimization. After using all the generators to the full capacity, the EMA uses storage and load shedding as options to match the load with generation in step 3.b of the proposed algorithm.

\section{NUMERICAL EXAMPLE}

To test the performance of the proposed EMS algorithm, a microgrid with a mix of definite energy sources (one fuel cell, one micro turbine and grid connection with upper limit of power drawl- whose maximum power availability is defined) and uncertain energy sources (one wind turbine and two photovoltaic panels) is considered. The details of the mentioned power generators are as tabulated in Table 1. The forecast of the maximum power available with the wind turbine, photovoltaic panel and the loads are as plotted in Figure 2. The sampling and scheduling is done on hourly basis for 24 hours. The dynamic prices of energy generation from fuel cell, micro turbine and the utility grid are as tabulated in Table 2. A battery of 300 $\mathrm{kW}$, which is optimum [30], approximately $15 \%$ of the microgrid capacity, is considered for back-up, whose technical details are as shown in Table 1. The DMO imposed upper limit for grid power drawl is fixed at $300 \mathrm{~kW}$.

Table 1. Technical Details of the generators used $(k W)$

\begin{tabular}{|l|l|l|l|l|}
\hline Type & Capacity & $\begin{array}{l}\text { Max power } \\
\text { limit }\end{array}$ & $\begin{array}{l}\text { Min power } \\
\text { limit }\end{array}$ & $\begin{array}{l}\text { Cost of energy } \\
(\text { Euro/kW) }\end{array}$ \\
\hline Wind tubine & 800 & 800 & -- & 0.0307 \\
\hline PV panels, 1 & 200 & 200 & -- & 0.0307 \\
\hline Fuel cell & 500 & 500 & 30 & As per Table-2 \\
\hline
\end{tabular}




\begin{tabular}{|l|l|l|l|l|}
\hline Micro turbine & 500 & 500 & 30 & As per Table-2 \\
\hline Grid & -- & 300 & -- & As per Table 2 \\
\hline Battery & 300 & 100 & Max SoC & \\
\hline & & & Min SoC & \\
\hline
\end{tabular}

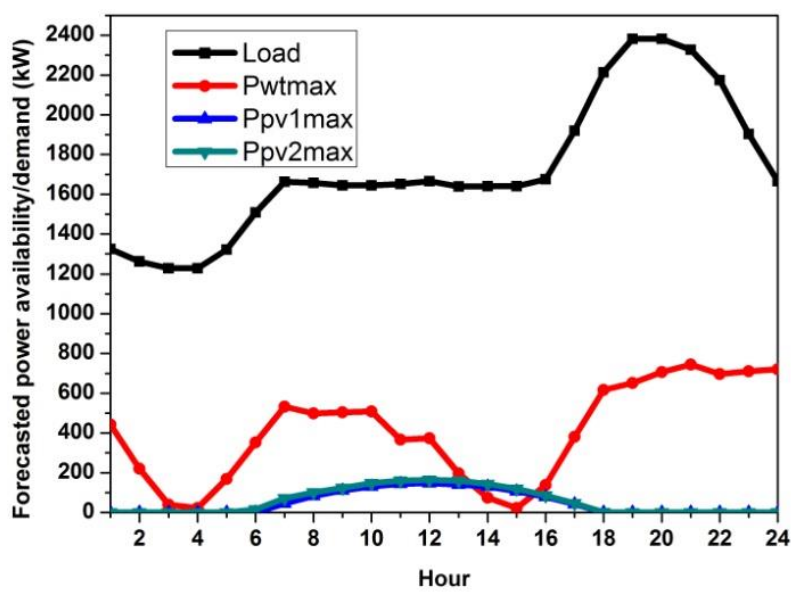

Figure 2. Forecasted power availability of RES generators and load

Table 2. Cost of energy in Euros/kWh

\begin{tabular}{|l|l|l|l|l|l|l|l|}
\hline Hour & $\mathrm{P}_{\text {grid }}$ & $\mathrm{P}_{\mathrm{mt}}$ & $\mathrm{P}_{\mathrm{fc}}$ & Hour & $\mathrm{P}_{\text {grid }}$ & $\mathrm{P}_{\mathrm{mt}}$ & $\mathrm{P}_{\mathrm{fc}}$ \\
\hline 1 & 0.027 & 0.0823 & 0.1277 & 13 & 0.572 & 0.0885 & 0.1308 \\
\hline 2 & 0.02 & 0.0831 & 0.1285 & 14 & 0.286 & 0.0885 & 0.138 \\
\hline 3 & 0.017 & 0.0831 & 0.129 & 15 & 0.279 & 0.09 & 0.1315 \\
\hline 4 & 0.017 & 0.0838 & 0.1285 & 16 & 0.086 & 0.0908 & 0.1331 \\
\hline 5 & 0.029 & 0.0838 & 0.1292 & 17 & 0.059 & 0.0915 & 0.1331 \\
\hline 6 & 0.033 & 0.0846 & 0.1292 & 18 & 0.05 & 0.0908 & 0.1338 \\
\hline 7 & 0.054 & 0.0854 & 0.13 & 19 & 0.061 & 0.0885 & 0.1331 \\
\hline 8 & 0.215 & 0.0862 & 0.1308 & 20 & 0.181 & 0.0862 & 0.1315 \\
\hline 9 & 0.572 & 0.0862 & 0.1315 & 21 & 0.077 & 0.0846 & 0.1308 \\
\hline 10 & 0.572 & 0.0892 & 0.1323 & 22 & 0.043 & 0.0838 & 0.13 \\
\hline 11 & 0.572 & 0.09 & 0.1315 & 23 & 0.037 & 0.0831 & 0.1285 \\
\hline 12 & 0.215 & 0.0885 & 0.1308 & 24 & 0.035 & 0.0832 & 0.1281 \\
\hline
\end{tabular}

To treat the uncertainty in maximum power availability from RES generators and hourly loads, several scenarios are generated using the uncertainty model explained in (19) and presented graphically in Figure 3 to Figure 6. The proposed energy management algorithm is implemented in scenario 3 using AFS algorithm and the results are tabulated in Table 4. The numeric data of scenario 3 is presented in Table 3 for easy comprehension. To initialize the parameters, the scheduled power of each generator (artificial fishes) is set to minimum power limit according to Table 1, the vision of the fishes is set to the maximum power limit of the generator and the step is set to $1 \mathrm{~kW}$ in common to all the generators. The results obtained using AFS algorithm are validated by comparing with the results obtained for implementing the same problem using AIMD algorithm. The results using AIMD algorithm are shown in Table 5. 


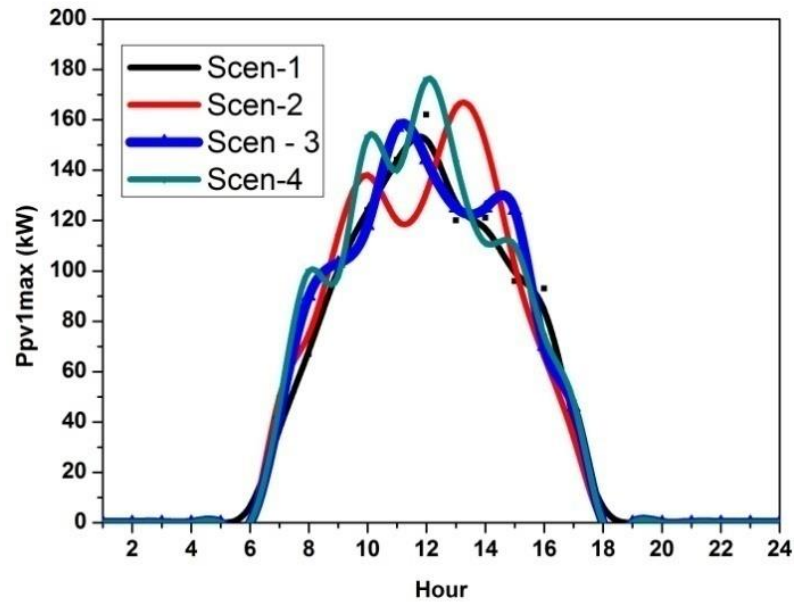

Figure 3. Scenarios for Max Power available with PVI

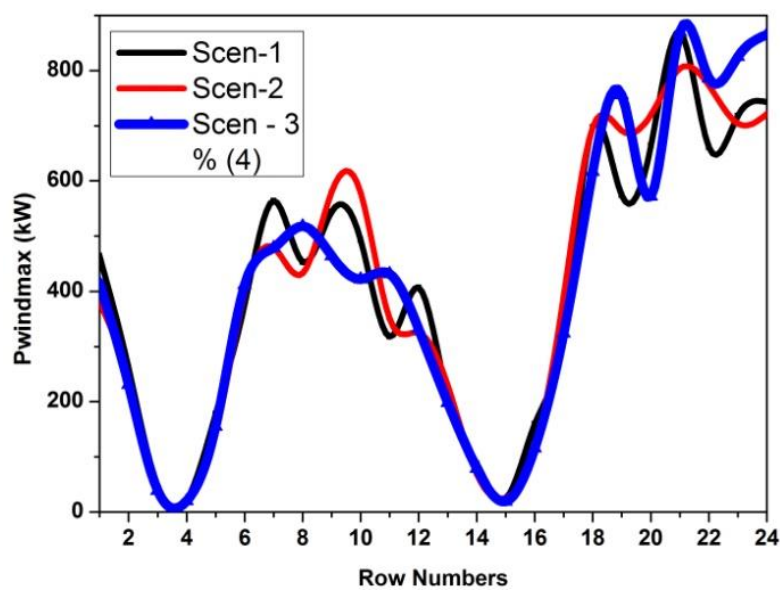

Figure 5. Scenarios for Max Power available with wind turbine

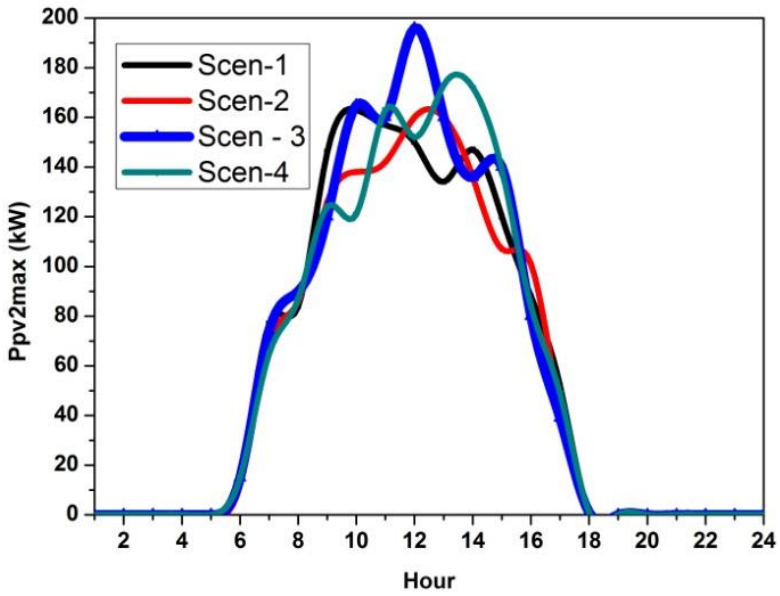

Figure 4. Scenarios for Max Power available with $P V 2$

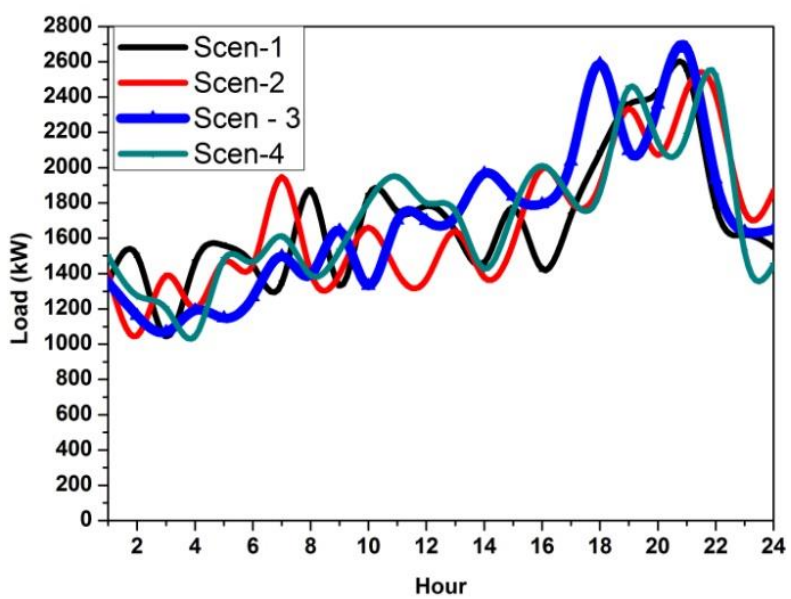

Figure 6. Scenarios for loads

Table 3. Power availability and load in uncertainty scenario $3(k W)$

\begin{tabular}{|c|c|c|c|c|c|c|c|c|c|}
\hline Hour & $\mathrm{P}_{\text {wind }}$ & $\mathrm{P}_{\mathrm{pv} 1}$ & $\mathrm{P}_{\mathrm{pv} 2}$ & load & Hour & $\mathrm{P}_{\text {wind }}$ & $\mathrm{P}_{\mathrm{pv} 1}$ & $\mathrm{P}_{\mathrm{pv} 2}$ & Load \\
\hline 1 & 415 & 0 & 0 & 1352 & 13 & 198 & 125 & 160 & 1721 \\
\hline 2 & 231 & 0 & 0 & 1162 & 14 & 79 & 125 & 136 & 1968 \\
\hline 3 & 39 & 0 & 0 & 1069 & 15 & 19 & 124 & 140 & 1837 \\
\hline 4 & 20 & 0 & 0 & 1192 & 16 & 116 & 70 & 80 & 1793 \\
\hline 5 & 155 & 0 & 0 & 1149 & 17 & 324 & 46 & 39 & 2035 \\
\hline 6 & 412 & 0 & 15 & 1268 & 18 & 617 & 0 & 1 & 2590 \\
\hline 7 & 479 & 43 & 74 & 1497 & 19 & 756 & 0 & 0 & 2096 \\
\hline 8 & 518 & 90 & 90 & 1392 & 20 & 572 & 0 & 0 & 2358 \\
\hline 9 & 464 & 103 & 120 & 1644 & 21 & 870 & 0 & 0 & 2676 \\
\hline 10 & 422 & 118 & 165 & 1332 & 22 & 786 & 0 & 0 & 1935 \\
\hline 11 & 432 & 157 & 160 & 1702 & 23 & 825 & 0 & 0 & 1637 \\
\hline 12 & 332 & 144 & 196 & 1699 & 24 & 865 & 0 & 0 & 1649 \\
\hline \multicolumn{6}{|c|}{ Total energy availability over 24 hours ( $\mathrm{kWh}$ ) } & 9946 & 1145 & 1376 & \\
\hline
\end{tabular}


Table 4. Scheduling results for scenario 3 using AFS algorithm (power in $k W$ and cost in Euros) \begin{tabular}{|l|l|l|l|l|l|l|l|l|l|l|l|}
\hline Hour & $\mathrm{Pg}_{\mathrm{wind}}$ & $\mathrm{Pg}_{\mathrm{pv} 1}$. & $\mathrm{Pg}_{\mathrm{pv} 2}$ & $\mathrm{Pg}_{\mathrm{fc}}$ & $\mathrm{Pg}_{\mathrm{mt}}$ & $\mathrm{P}_{\text {grid }}$ & $\mathrm{P}_{\text {disch }}$ & Cost & $\mathrm{P}_{\text {charge }}$ & SoC & Loadshed \\
\hline
\end{tabular}

\begin{tabular}{|l|l|l|l|l|l|l|l|l|l|l|l|l|}
\hline 1 & 415 & 0 & 0 & 237 & 500 & 300 & 0 & 92.26 & 100 & 130 & 0 \\
\hline 2 & 231 & 0 & 0 & 220 & 481 & 300 & 0 & 81.33 & 70 & 230 & 0 \\
\hline 3 & 36 & 0 & 0 & 240 & 500 & 293 & 0 & 78.6 & 0 & 300 & 0 \\
\hline 4 & 5 & 0 & 0 & 399 & 488 & 300 & 0 & 97.42 & 0 & 300 & 0 \\
\hline 5 & 151 & 0 & 0 & 224 & 499 & 275 & 0 & 83.37 & 0 & 300 & 0 \\
\hline 6 & 389 & 0 & 8 & 153 & 448 & 270 & 0 & 78.77 & 0 & 300 & 0 \\
\hline 7 & 472 & 8 & 70 & 189 & 477 & 281 & 0 & 97.36 & 0 & 300 & 0 \\
\hline 8 & 495 & 79 & 54 & 205 & 499 & 60 & 0 & 102.01 & 0 & 300 & 0 \\
\hline 9 & 423 & 51 & 116 & 498 & 488 & 68 & 0 & 164.56 & 0 & 300 & 0 \\
\hline 10 & 415 & 102 & 106 & 230 & 470 & 9 & 0 & 96.63 & 0 & 300 & 0 \\
\hline 11 & 412 & 126 & 132 & 459 & 489 & 84 & 0 & 172.99 & 0 & 300 & 0 \\
\hline 12 & 312 & 144 & 119 & 440 & 491 & 192 & 0 & 160.15 & 0 & 300 & 0 \\
\hline 13 & 198 & 125 & 160 & 500 & 500 & 238 & 0 & 260.61 & 0 & 300 & 0 \\
\hline 14 & 79 & 125 & 136 & 500 & 500 & 300 & 100 & 209.49 & 0 & 300 & 228 \\
\hline 15 & 19 & 124 & 140 & 500 & 500 & 300 & 100 & 203.14 & 0 & 200 & 154 \\
\hline 16 & 116 & 70 & 80 & 500 & 500 & 300 & 70 & 145.92 & 0 & 100 & 157 \\
\hline 17 & 324 & 46 & 39 & 500 & 500 & 300 & 0 & 142.56 & 0 & 30 & 326 \\
\hline 18 & 617 & 0 & 1 & 500 & 500 & 300 & 0 & 146.27 & 0 & 30 & 672 \\
\hline 19 & 756 & 0 & 0 & 500 & 500 & 300 & 0 & 152.31 & 0 & 30 & 40 \\
\hline 20 & 572 & 0 & 0 & 500 & 500 & 300 & 0 & 180.71 & 0 & 30 & 486 \\
\hline 21 & 870 & 0 & 0 & 500 & 500 & 300 & 0 & 157.51 & 0 & 30 & 506 \\
\hline 22 & 786 & 0 & 0 & 486 & 463 & 300 & 0 & 139.01 & 100 & 30 & 0 \\
\hline 23 & 820 & 0 & 0 & 137 & 480 & 300 & 0 & 93.77 & 100 & 130 & 0 \\
\hline 24 & 836 & 0 & 0 & 160 & 423 & 300 & 0 & 91.85 & 70 & 230 & 0 \\
\hline
\end{tabular}

Table 5. Scheduling results for scenario 3 using AIMD algorithm (powers in $k W$ and cost in Euros)

\begin{tabular}{|l|l|l|l|l|l|l|l|l|l|l|l|}
\hline Hour & $\mathrm{Pg}_{\text {wind }}$ & $\mathrm{Pg}_{\mathrm{pv} 1}$. & $\mathrm{Pg}_{\mathrm{pv} 2}$ & $\mathrm{Pg}_{\mathrm{fc}}$ & $\mathrm{Pg}_{\mathrm{mt}}$ & $\mathrm{P}_{\text {grid }}$ & $\mathrm{P}_{\text {disch }}$ & Cost & $\mathrm{P}_{\text {charg }}$ & SoC & Loadshed \\
\hline 1 & 364 & 0 & 0 & 394 & 394 & 300 & 0 & 102.01 & 100 & 130 & 0 \\
\hline 2 & 231 & 0 & 0 & 350.5 & 350.5 & 300 & 0 & 87.26 & 70 & 230 & 0 \\
\hline 3 & 39 & 0 & 0 & 365 & 365 & 300 & 0 & 83.71 & 0 & 300 & 0 \\
\hline 4 & 20 & 0 & 0 & 436 & 436 & 300 & 0 & 98.28 & 0 & 300 & 0 \\
\hline 5 & 155 & 0 & 0 & 347 & 347 & 300 & 0 & 87.37 & 0 & 300 & 0 \\
\hline 6 & 298.25 & 0 & 15 & 328.25 & 328.25 & 298.25 & 0 & 89.64 & 0 & 300 & 0 \\
\hline 7 & 340 & 43 & 74 & 370 & 370 & 300 & 0 & 109.93 & 0 & 300 & 0 \\
\hline 8 & 288 & 90 & 90 & 318 & 318 & 288 & 0 & 145.29 & 0 & 300 & 0 \\
\hline 9 & 353.68 & 103 & 120 & 383.67 & 383.67 & 299.98 & 0 & 272.82 & 0 & 300 & 0 \\
\hline 10 & 247.26 & 117.98 & 165 & 277.25 & 277.25 & 247.25 & 0 & 219.12 & 0 & 300 & 0 \\
\hline 11 & 341.68 & 157 & 160 & 371.67 & 371.67 & 299.98 & 0 & 274.13 & 0 & 300 & 0 \\
\hline 12 & 332 & 144 & 196 & 363.5 & 363.5 & 300 & 0 & 167.85 & 0 & 300 & 0 \\
\hline 13 & 198 & 125 & 160 & 469 & 469 & 300 & 0 & 289.28 & 0 & 300 & 0 \\
\hline 14 & 79 & 125 & 136 & 500 & 500 & 300 & 100 & 209.49 & 0 & 300 & 228 \\
\hline 15 & 19 & 124 & 140 & 500 & 500 & 300 & 100 & 203.14 & 0 & 200 & 154 \\
\hline 16 & 116 & 70 & 80 & 500 & 500 & 300 & 70 & 145.92 & 0 & 100 & 157 \\
\hline 17 & 324 & 46 & 39 & 500 & 500 & 300 & 0 & 142.56 & 0 & 30 & 326 \\
\hline 18 & 617 & 0 & 1 & 500 & 500 & 300 & 0 & 146.27 & 0 & 30 & 672 \\
\hline 19 & 756 & 0 & 0 & 500 & 500 & 300 & 0 & 152.31 & 0 & 30 & 40 \\
\hline 20 & 572 & 0 & 0 & 500 & 500 & 300 & 0 & 180.71 & 0 & 30 & 486 \\
\hline
\end{tabular}




\begin{tabular}{|l|l|l|l|l|l|l|l|l|l|l|l|}
\hline 21 & 870 & 0 & 0 & 500 & 500 & 300 & 0 & 157.51 & 0 & 30 & 506 \\
\hline 22 & 735 & 0 & 0 & 500 & 500 & 300 & 0 & 142.36 & 100 & 30 & 0 \\
\hline 23 & 459 & 0 & 0 & 489 & 489 & 300 & 0 & 128.66 & 100 & 130 & 0 \\
\hline 24 & 453 & 0 & 0 & 483 & 483 & 300 & 0 & 126.47 & 70 & 230 & 0 \\
\hline
\end{tabular}

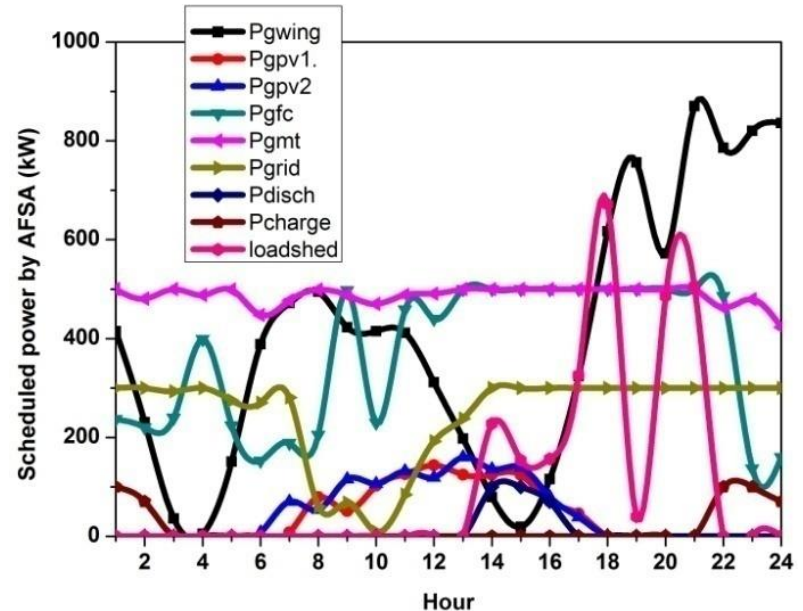

Figure 7. Scheduling results using AFS algorithm

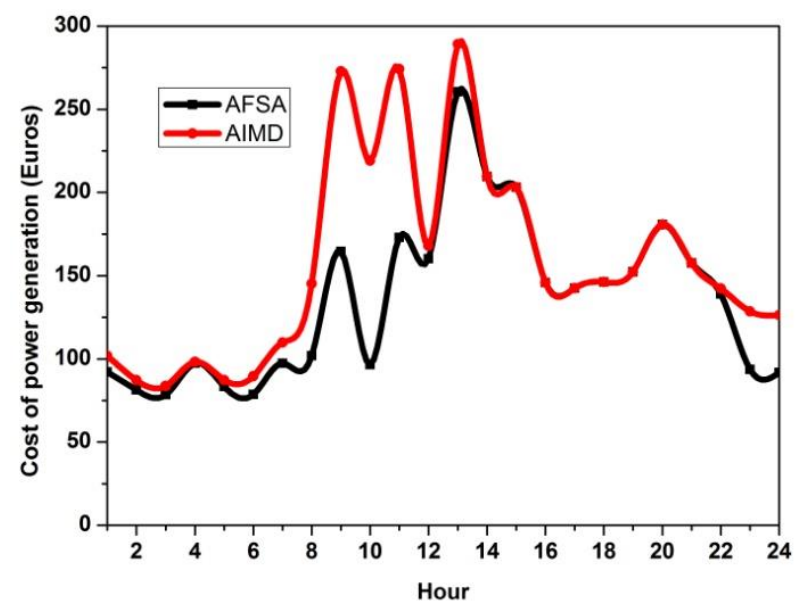

Figure 9. Comparison of cost of generation using AFS and AIMD algorithms

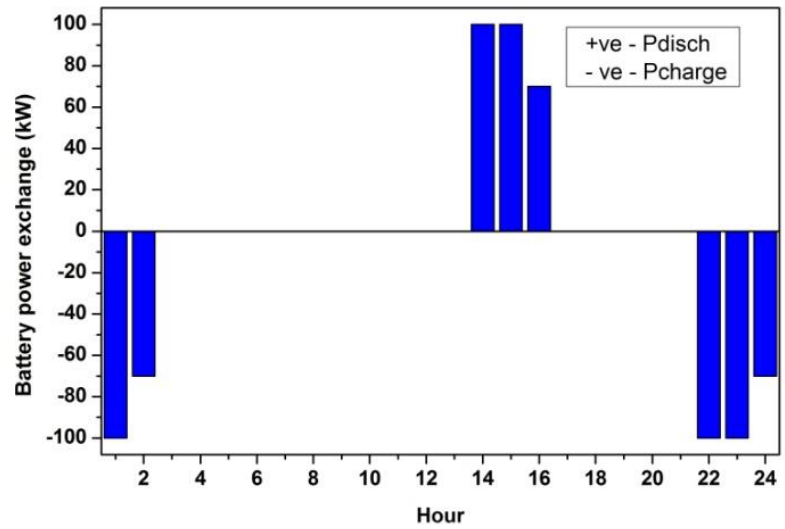

Figure 10. Battery power exchange

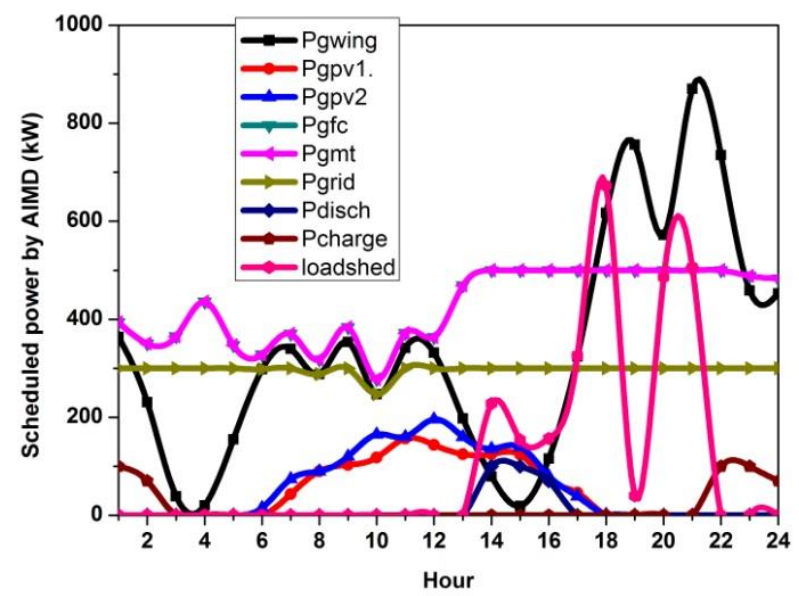

Figure 8. Scheduling results using AIMD algorithm 


\section{RESULTS AND DISCUSSIONS}

The results of generation scheduling by implementing proposed algorithm for scenario-3 is presented in Table 4 and that using AIMD algorithm in Table 5. The term Pg with suffix in the Tables 4 and 5 refers to the power generation scheduled to the respective generators after implementing the proposed Energy Management Algorithm. In either case, the fundamental constraint of energy balance as laid down by (19) is verified and the power produced (Power produced=Power generated by conventional generators+Power generated by renewable generators+Power obtained by battery discharge+Power drawn from grid, (22)] is exactly matching the power required [Power required = Total load on microgrid-Load shed+Powre required to charge battery, (22)] at each hour. A close comparison of Table 4 and Table 5 shows that AFS algorithm is far better than AIMD algorithm in optimizing the cost of generation. For example at hour 1, the cost of grid energy is the cheapest, followed by wind and PV power, micro turbine and fuel cell in sequence of increasing cost of generation. During this hour, the proposed EMS using AFS algorithm is able to schedule complete power available from cheaper sources (grid, wind turbine, PV panels, micro turbine) and use the costliest source, i.e., fuel cell very sparingly. Whereas AIMD algorithm is unable to do the same. It is scheduling equal powers to all the generators subject to min and max powers. For micro turbine and fuel cell, the min powers are $30 \mathrm{~kW}$ (Table 1) and for wind turbine and PV panels, the min powers are zero. Max powers available for wind turbine and PV panels are $415 \mathrm{~kW}, 0 \mathrm{~kW}, 0 \mathrm{~kW}$ (Table-3, uncertainty scenario 3) during hour 1 and for micro turbine, fuel cell and grid it is $500 \mathrm{~kW}, 500 \mathrm{~kW}$ and $300 \mathrm{~kW}$ respectively (Table 1). At hour 1, AIMD algorithm is allocating equal power scheduling to wind (364 $\mathrm{kW})$, fuel cell $(394-30(\min$ power $)=364 \mathrm{~kW})$ and micro turbine $(394-30(\min$ power $)=364 \mathrm{~kW})$ and $300 \mathrm{~kW}$ to grid as its maximum power limit is $300 \mathrm{~kW}$. In other words, the AFS algorithm is able to choose the cheaper sources and schedule more load to them compared to the costlier sources, whereas AIMD algorithm is scheduling equal amounts of loads to all the sources available irrespective of their cost of energy generation and that is the reason why there is a difference in cost of energy generation estimated by the two algorithms. Similar scheduling is done for hours 1-13 and hours 22-24. During these hours, the demand is less than the total power availability and hence the AFS algorithm is able to search for the most economic mix of power generation from different sources. During the hours $14-21$, the demand is more than the total power available, and hence both the algorithms are using entire power available, there is no choice of selection of generators for scheduling and hence the cost estimation by both the algorithms is same. The above discussion compares the performance of two tools used for optimization, i.e., AIMD algorithm and AFS algorithm. Figure 7 and Figure 8 show the scheduling results using two algorithms. Figure 9 compares the costs of energy generation estimated by the two optimization tools. As far as the performance of the Energy Management System proposed, it is able to balance the power required with the power generation at each hour. The battery is assumed to be at min state of charge $(30 \mathrm{~kW})$ at the beginning of hour 1 . The EMS is able to charge the battery to its maximum state of charge without violating the maximum rate of charge, i.e., $100 \mathrm{~kW}$, during the hours when excess generation is available after supplying the load. Similarly it is able to discharge the battery when required, i.e., during hours 14,15 and 16 without violating the maximum discharge rate, i.e., $100 \mathrm{~kW}$. Figure 10 shows the hourly power exchange of the battery on hourly basis. The proposed EMS is also able to maintain the state of charge of the battery within the upper and lower limits as mentioned in Table 1. Figure 11 shows the hourly state of charge of the battery over the day. The EMS is also able to implement load shedding during deficit generation hours, after discharging the battery. During the peak hours, i.e., between hours 14-21, the EMS is implementing load shedding to balance the power required with power generated.

\section{CONCLUSIONS}

An energy management system for day ahead energy planning in a microgrid is proposed in this article with an objective to optimize the energy generation cost and to reduce the effect of uncertainty due to renewable sources. The proposed EMS is tested on a numerical problem in a grid connected microgrid with several hybrid sources like a fuel cell, a micro turbine, PV and wind generators along with battery backup under dynamic pricing policy. Several uncertainty scenarios are drawn for power availability from renewable generators and the proposed EMS is applied for day ahead scheduling considering the $3^{\text {rd }}$ scenario. AFS algorithm which is used as a tool to solve the optimization part, has estimated the total generation cost over a day as 3228.9 Euros as against AIMD algorithm, which estimated the same as 3762 
Euros. The AFS algorithm is better optimizing the generation mix with a net saving of $16.5 \%$. The proposed EMS able to balance the generation with load at each interval. The main objective of the proposed EMS is reducing the effect of uncertainty of renewable energy generation and it is well attained using battery storage. The EMS is able to schedule battery storage during excess generation and discharge the battery during deficit generation while maintaining the rate of power exchange and state of charge limits. The EMS is also able to implement load shedding when there is no possibility to supply the demand in full during the peak hours, i.e., between $14^{\text {th }}$ to $21^{\text {st }}$ hours.

\section{CONFLICTS OF INTEREST}

No conflict of interests was declared by the authors.

\section{REFERENCES}

[1] https://www.morganstanley.com/ideas/clean-energy-trump.html. (accessed on 25.12.2018).

[2] http://economictimes.indiatimes.com/industry /energy/ power/ solar-power-tariff-drops-tohistoric-low-at-rs-2-44-per-nit/articleshow/58649942.cm,(accessed on 25.12.2018).

[3] K. Prakash Kumar, B. Saravanan, "Recent techniques to model uncertainty in power generation from renewable energy sources in microgrids", J. Renew Sustain Energy Rev, 71, 348-58, (2017).

[4] Basu Ashoke Kumar, Chowdhury SP, Chowdhury S, Paul S. "Microgrids: energy management by strategic deployment of DERs-A comprehensive review", Renew Sustain Energy Rev 15, 434856, (2011).

[5] El Bakari K, Kling WL., "Virtual power plant: Answer to increasing distributed generation", IEEE Proc, PES conf Innov Smartgrid Technol (Eur), 11-13, 1-6, (2010).

[6] K. Prakash Kumar, B. Saravanan and K.S.Swarup, "A two stage increase decrease algorithm to optimize distributed generation in a virtual power plant", Energy Procedia, 90, 276-82, (2016).

[7] GE Energy . Western wind and solar integration study [tech rep]. NREL (2010).

[8] Hawkes AD, Leach MA. "Modelling high level system design and unit commitment for a microgrid", Appl Energy, 86, 1253-65, (2009).

[9] Van der Kam M, van Sark W. "Smart charging of electric vehicles with photovoltaic power and vehicle-to-grid technology in a microgrid; a case study", Appl Energy, 152, 20-30, (2015).

[10] Zhang Z, Wang J, Wang X. "An improved charging/discharging strategy of lithium batteries considering depreciation cost in day-ahead microgrid scheduling", Energy Convers Manage, 105, 675-84, (2015).

[11] Mallol-Poyato R, Salcedo-Sanz S, Jimenez-Fernandez S, Diaz-Villar P. "Optimal discharge scheduling of energy storage systems in MicroGrids based on hyperheuristics", Renew Energy, 83, 13-24, (2015).

[12] Thillainathan Logenthiran, Dipti Srinivasan, Tan Zong Shun, "Demand side management in smartgrid using heuristic optimization”, IEEE transaction on Smartgrid, 3, no.3, (2012).

[13] Zakariazadeh Alireza, Jadid Shahram, Siano Pierluigi. "Smart microgrid energy and reserve scheduling with demand response using stochastic optimization", Electr Power Energy Syst, 63, 523-33, (2014). 
[14] Montuori L, Alcazar-Ortega M, Alvarez-Bel C, Domijan A. "Integration of renewable energy in microgrids coordinated with demand response resources: economic evaluation of a biomass gasification plant by Homer Simulator", Appl Energy, 132, 15-22, (2014).

[15] Mazidi M, Zakariazadeh A, Jadid S, Siano P. "Integrated scheduling of renewable generation and demand response programs in a microgrid", Energy Convers Manage, 86, 1118-27, (2014).

[16] Mohammadreza Mazidi, Hassan Monsef,Pierluigi Siano, "Robust day-ahead scheduling of smart distribution networks considering demand response programs", Applied Energy 178 (2016) 929942, (2016).

[17] Cherukuri, S. Hari Charan, and B. Saravanan. "A novel energy management algorithm for reduction of main grid dependence in future smart grids using electric springs." Sustainable Energy Technologies and Assessments 21, 1-12, (2017).

[18] Talari S, Yazdaninejad M, Haghifam M. "Stochastic-based scheduling of the microgrid operation including wind turbines, photovoltaic cells, energystorages and responsive loads", IET Gener Transm Distrib, 9, 1498-509, (2015).

[19] Najibi F, Niknam T. "Stochastic scheduling of renewable micro-grids considering photovoltaic source uncertainties", Energy Convers Manage, 98, 484-99, (2015).

[20] Najibi F, Niknam T. "Stochastic scheduling of renewable micro-grids considering photovoltaic source uncertainties", Energy Convers Manage, 98, 484-99, (2015).

[21] Zakariazadeh A, Jadid S, Siano P. "Smart microgrid energy and reserve scheduling with demand response using stochastic optimization”, Int J Electr Power Energy Syst, 63, 523-33, (2014).

[22] Yu Zhang, NikolaosGatsis, and Georgios B. Giannakis, "Robust Energy Management for Microgrids With High-Penetration Renewables", IEEE Transactions on Sustainable energy, 4, No.4, (2013).

[23] Amin Khodaei, "Microgrid Optimal Scheduling With Multi-Period Islanding Constraints, IEEE Trasactions on power systems", 29,No.3, (2014).

[24] Emmanual Cristoni, Marco Raugi and Robert Shorten (2014), "Plug and Play distributed algorithm for Optimized power Generation in a Microgrid", IEEE Transactions on Smart Grid, 4, 2145-54, (2014).

[25] Yaowang Li, Shihong Miao, Xing Luo, Jihong Wang, "Optimization scheduling model based on source-load-energy storage coordination in power systems", International Conference on Automation and Computing. IEEE, 120-125, (2016).

[26] Zhigang Li, Wenchuan Wu, Boming Zhang, "Adjustable robust real-time power dispatch with large-scale wind power integration”, IEEE Trans. Sustain. Energy, 6 (2), 357-68, (2015).

[27] Bo Xing, Wen Jing Gao, "Innovative computational intelligence: A rough guide to 134 clever algorithms", Springer International Publishing, Switzerland, (2014).

[28] K. Prakash Kumar, B. Saravanan, "Day ahead scheduling of generation and storage in a microgrid considering demand side management", Journal of Energy Storage, 21, 78-86, (2019).

[29] Sina Parhizi, Amin Khodaei, Mohammad Shahidehpour, "Market based verses Price based microgrid optimal scheduling", IEEE Trans on Smartgrids, 9(2), 615-23, (2018). 
[30] Braun B, Philipp B, Swierczynski S, Jozef MM, Diosi D, Robert R, Stroe S, Loan D. Teodorescu $\mathrm{T}$, Remus R. "Optimizing a hybrid energy storage system for a virtual power plant for improved wind power generation: A case study for Denmark", Proceedings of the 6th International Renewable Energy Storage Conference and Exhibition, IRES, 1-9, (2011).

\section{APPENDIX}

\section{Abbreviations}

$P_{i}^{\text {con }}(t)$

$P_{j}^{r e s}(t)$

$P_{i}^{\text {con }_{\max }}, P_{i}^{\text {con }_{\min }}$

$P_{j}^{r e s_{\max }}, P_{j}^{\text {res }}$

$W_{i}^{\text {con }}(t), W_{j}^{r e s}(t)$

$P_{k}^{s t g_{\text {ch }} \text { max }}, P_{k}^{\text {stg } \text { dch }_{\text {max }}}$

$\operatorname{SoC}_{k}(t)$

$\operatorname{SoC}_{k}^{\max }, \operatorname{SoC}_{k}^{\min }$

$\eta_{k}$

$L_{m}^{\text {shd }}(t)$

$C_{m}^{\text {shd }}(t)$

$Y_{m}^{\text {shd }}(t)$

$C_{i}^{c o n}(t)$

$C_{j}^{r e s}(t)$

$c_{i}^{c o n}(t)$

$c_{i}^{\text {con }}{ }_{\text {start }}, c_{i}^{\text {con }}$ shut

$U_{i}^{\text {con }}(t), V_{i}^{\text {con }}(t)$

$U_{j}^{\text {res }}(t), V_{j}^{\text {res }}(t)$

$X_{i}^{\text {con }}(t), X_{j}^{r e s}(t)$

$C_{k}^{\text {stg }}(t)$

$c_{k}^{s t g}(t)$

$Y_{k}^{s t g}(t)$

$C^{\text {grd }}(t)$

$c^{\text {grd }}(t)$

$P^{\text {grd }}(t)$

$\Psi_{j}$

$\psi$
Power generated by $i^{t h}$ generator at time interval $t$.

Power generated by $j^{\text {th }}$ RES generator at time interval $t$.

Maximum and Minimum power limits of $\mathrm{i}^{\text {th }}$ generator at time interval $t$.

Maximum and Minimum power limits of $j^{\text {th }}$ RES generator at time interval $t$.

State vectors denoting ON/OFF states of $i^{\text {th }}$ generator and $j^{\text {th }}$ RES generator at time interval $t$ respectively.

Maximum Charge/Discharge rates of $k^{\text {th }}$ storage device.

State of charge (SoC) of $k^{t h}$ storage device at time interval $t$.

Maximum and minimum limits of SoC of $k^{\text {th }}$ storage device.

Efficiency (Discharging/Charging) of $k^{\text {th }}$ storage device.

$m^{\text {th }}$ load shed at time interval $t$.

Compensation due to shedding of $m^{\text {th }}$ load at time interval $t$.

State of vector denoting Connected/Shed state of $m^{\text {th }}$ load at time interval $t$.

Power generation cost of $i^{\text {th }}$ generator at time interval $t$.

Power generation cost of $j^{\text {th }}$ RES generator at time interval $t$.

Cost/unit of power generation of $i^{t h}$ conventional generator at time $t$.

Start-up and shut-down cost of $i^{\text {th }}$ conventional generator.

State vectors denoting the shut-down/start-up states of $i^{\text {th }}$ generator at time interval $t$.

State vectors denoting the shut-down/start-up states of $j^{\text {th }}$ renewable generator at time interval $t$.

State vectors denoting the OFF/ON position of the generators and RES at time $t$.

Expenses of energy supply by $k^{t h}$ storage device at time interval $t$.

Unit cost energy supply from $k^{t h}$ storage device at time interval $t$.

State vector denoting the type of power exchange by $k^{\text {th }}$ storage device at time interval $t$.

Power drawing expenses from grid at time interval $t$.

Unit cost of grid power at time interval $t$.

Power drawn from grid at time interval $t$.

Vector denoting all numeric values of $P_{j}^{r e s}(t)$.

Uncertainty set. 
$P_{j, s}^{\text {restotal }_{\min }}, P_{j, s}^{\text {restotal }}$ max Upper and lower limits for total energy availability with the $j^{\text {th }}$ RES over a time subhorizon S.

\section{$t$}

$P_{i}(t)$

$d(t)$

$P_{i_{\max }}, P_{i_{\min }}$
Time interval.

Power generated by $i^{\text {th }}$ generator at time interval $t$.

Demand at time interval $t$.

Generation limits of $i^{\text {th }}$ generator. 